\title{
A Theoretic Review of Emotion Regulation
}

\author{
Hong Chen \\ School of management, Jinan University, Guangzhou, China \\ Email: ch123xj@163.com
}

Received 29 January 2016; accepted 22 February 2016; published 25 February 2016

Copyright (C) 2016 by authors and Scientific Research Publishing Inc.

This work is licensed under the Creative Commons Attribution International License (CC BY).

http://creativecommons.org/licenses/by/4.0/

(c) (i) Open Access

\begin{abstract}
Emotion regulation has become a hot research topic in psychological research in recent years; this study reviewed the research on emotion regulation strategies in the last ten years, and the previous research focused on introducing a new concept of regulation strategy-reception strategy, and to explore the similarities and differences between the emotional response and the neural mechanism of cognitive assessment and the expression of inhibitory strategies. It was found that cognitive assessment could reduce the emotional experience, reduce the activation of the physiological response and sympathetic nervous system, and decrease the level of activation of the amygdala; while the inhibition of expression was able to reduce emotional behavior, but the physiological response and sympathetic nervous system activation was enhanced, and the level of activation of the amygdala did not decrease; at the same time, it was a kind of emotion regulation strategy between cognitive reappraisal and expression inhibition, the regulation effect of the emotion was better than that of the expression inhibition, but less than the cognitive reappraisal, in the neural mechanism, the three common brain areas.
\end{abstract}

\section{Keywords}

Emotion Regulation Strategy, Reception Strategy, Cognitive Reappraisal, Inhibition of Expression

\section{Introduction}

The study of human emotion has a long history, and the influence of emotion on human cognition and decision making process has been widely explored and measured by psychologists. With the deepening of research, emotion regulation has become one of the hot issues in the study of psychology. In the study of cognitive psychology, it is found that different emotion regulation strategies can affect the memory and the distribution of attention [1] [2]. Since Gross put forward the model of emotion regulation process and strategy theory [1] [3], researchers at home and abroad have carried out experiments and measurement research on emotion regulation strategies. In the model of emotion regulation process, the two regulatory strategies of cognitive reappraisal and expression 
inhibition by Gross have been widely studied. At the same time, with the orientation of psychotherapy (Acceptance-oriented Therapy) receives more and more attention and evidence support, and it is known as the third wave of clinical behavior therapy [4]. The common basis of these accepted orientations of psychotherapy-accepted strategy as a kind of emotion regulation strategy begins to receive extensive attention and a lot of research. Thus, compared with the traditional emotion regulation strategy (Cognitive reappraisal and expression repression), accepted strategy gradually becomes a new emotion regulation strategy and becomes a new research method [5] [6]. A large number of studies have explored and measured the different effects of these three kinds of emotion regulation modes on emotion, cognition and social behavior. Based on the review and integration of these studies, this study focuses on the comparison of these three strategies in emotional experience and physiological responses and activation of different brain regions, to find cognitive reappraisal, expression inhibition and accepted strategy, which is better in the process of emotion regulation, and from the perspective of the brain mechanism to be revealed.

\section{Emotion and Emotion Regulation}

Emotion is a state of being aroused and experienced by the individual, sometimes emotional arousal is conscious, sometimes it is unconscious [7]. Gross thinks that emotion is an individual to deal with the important challenges and opportunities arising from a coordinated behavior, experience and physiological reaction tendency [8]. Most of time, the individual's emotional reaction is consistent with the change of living environment. But sometimes the individual's emotions cannot keep up with the pace of the times, this time the emotions will mislead us. In the case of this kind of emotion and the situation is not consistent, individuals need to adjust the mood to adapt to the environment changes.

\subsection{Emotion Regulation}

Gross thinks emotion regulation is the process of individual for what kind of emotions, when emotions appear, how to affect the expression of emotional experience and expression [9]. In simple terms, Emotion regulation is the process of individual management and change of oneself or other people's emotions. In this process, through a certain strategy and mechanism, causes the emotion in the physiological activity, the subjective experience, the expression behavior and so on the aspect to have the certain change [10]. Thus, the emotion regulation involves the process of the change of the latent period of emotion, the occurrence time, the duration, the behavior expression, the psychological experience, the physiological reaction and so on. This is a dynamic process.

\subsection{Emotion Regulation Strategy}

\subsubsection{Traditional Emotion Regulation Strategies: Cognitive Assessment and Expression Inhibition} Gross based on the emotional regulation occurs in the emotional reaction before or after the emotional reaction, emotional regulation is divided into two areas-Antecedent-focused emotion regulation and Response-focused emotion regulation. Antecedent-focused emotion regulation means select scenarios, modify scenarios, pay attention to assignments, and cognitive changes occur before the activation of the emotional response. Response-focused emotion regulation means the reaction adjustment occurred in the mood has been formed, after the activation of emotional response, therefore, the reaction is concerned about emotional regulation.

Gross propose, in the whole process of emotion, individuals have a lot of emotional regulation strategy. But the most common and valuable strategies to reduce emotional responses have two types. They are cognitive reappraisal and expression suppression. Cognitive reappraisal is cognitive change, change the understanding of emotional events, and change the personal meaning of emotional events. Cognitive reappraisal is the premise of the emotional regulation strategy. Inhibition of expression is a kind of reaction adjustment, it is the emotion regulation strategy which is concerned by inhibiting the expression of emotion that is going to happen or is happening. The expression inhibition transfers the self-control ability, starts the self-control process to restrain oneself mood behavior. Gross conducted a lot of experiments and measurements; it is found that two different emotion regulation strategies have different effects on emotion, cognition and social behavior [11].

\subsubsection{Reception Strategy}

Reception Strategy suggest do not make unnecessary to try to change the frequency or the form of personal emotional events, especially when this may cause adverse effects on mental. The reason why accept strategy as 
an emotion regulation strategy in emotion regulation in the field caused widespread concern, and accept orientation therapy in the treatment of emotional and other psychological problems (depression, anxiety, trauma, stress, burnout and chronic pain and addiction, etc.) achieved results have a close relationship [6]. Numerous orientation of psychological therapy is based on such a profit based: mental illness patients by excessive regulation to avoid the tendency to experience negative emotions that excessive regulation will play the opposite role. Under such background, in recent years, many researchers have accepted as a kind of emotion regulation strategy into the field of emotion regulation.

\section{A Comparison of the Effects of Cognitive Assessment, Expression Inhibition and Reception Strategy on Emotion}

\subsection{Comparison of Cognitive Reappraisal and Expression Inhibition on Emotion Regulation}

The main goal of emotion regulation is to revise the emotional reaction, and the specific strategies of the two different ways of emotion regulation have different effects on the subsequent emotion. According to the process model of emotion regulation, Gross is assumed to regulate emotion through cognitive reappraisal, which will change the locus of the whole emotional response, and reduce the psychological experience, behavioral expression and physiological reaction of emotion. And the expression inhibition reduced the expression behavior, but it will not reduce the emotional psychological experience, even the physiological responses of emotion can be enhanced by inhibiting the expression of emotional behavior, a large number of experiments and measurement studies conducted by Gross also support the above construction.

\subsubsection{The Impact on Emotional Experience: Compared with Expression Suppression, Cognitive Reappraisal Can Reduce Emotional Experience}

In order to test the effect of cognitive reappraisal on emotional responses, there are two kinds of orientation: guidance of cognitive review and spontaneous cognitive reappraisal. The guidance of cognitive reappraisal is to use the language of instruction to require the use of re-evaluation strategies to regulate emotions. The study found that subjects were asked to watch the aversion of movie clips, cognitive reappraisal reduced the aversion behavior expression, reduced the aversion of the experience, and expression suppression reduced the aversion behavior expression, but the emotional experience did not significantly affect [12]. In the study of spontaneous emotion regulation strategies, the differences of emotional experience between individuals using different strategies were mainly investigated [13]. Using emotion regulation questionnaire to examine the effect of emotion regulation on the individual with cognitive reappraisal, These studies show that individuals who are accustomed to using cognitive strategies of re-evaluation, negative emotional experience is low, and the habit of using suppression strategies to experience more negative emotions [14].

At the same time, Gross and Levenson further verified the different results of positive and negative expression of different emotional behavior, it was found that there was no significant effect on the emotion experience of negative emotion (such as sadness and disgust), but positive emotions (happiness, pride, etc.) suppression, its emotional experience has significant impact. That is to inhibit the positive emotional expression, leading to the positive emotional experience of the report is reduced [15].

In summary, cognitive assessment and inhibition of expression can effectively reduce the expression of emotional behavior, but the comparison between the two, cognitive reappraisal can better reduce the emotional experience.

\subsubsection{Effects on Physiological Responses: Inhibition of Expression and Enhancement of Emotional and Physiological Responses, Cognitive Reappraisal of the Weakening of the Emotional and Physiological Responses}

In the study of positive and negative emotions, there were no significant differences in the expression of behavioral responses. But on the contrary, the expression of inhibition and enhancement of emotional and physiological responses, cognitive reappraisal of the weakening of the emotional physiological reaction [12] [13]. More specific research reveals the differences between the two: using cognitive reappraisal strategy, caused by the strong side of the sympathetic nervous system activation, and reduce the heart rate, skin conductance level and the respiratory frequency, of the sympathetic nervous system in cardiovascular decreased activation [16]. 
Domestic and the use of physiological experiment method to study different methods to regulate the negative emotional changes in the process, the results show that the use of expression suppression, the finger pulse amplitude greater pulse [17].

In conclusion, the expression inhibition and cognitive reappraisal two kinds of emotion regulation strategies on the physiological reaction of their different: inhibition of expression of enhanced emotional physiological reaction, cognitive reappraisal weakened emotional physiological reaction.

\subsection{Comparison the Regulation of Reception Strategies and Cognitive Reappraisal and Expression Inhibition on Emotion}

Accepted strategy, as a new kind of emotion regulation strategy which is gradually accepted by the beginning of the study of emotion regulation in the field of psychotherapy. Current research is mainly concerned with the improvement of the negative emotions of anxiety and depression in patients with specific emotional disorders in clinical practice [5] [18]. Campbell-Sills and colleagues compared the effects of two aspects of acceptance and expression inhibition on anxiety, respectively, from two aspects of the spontaneous emotion regulation strategy and the guidance of the emotional regulation. Campbell-Sills in the experiment to the experimental group (anxiety and mood disorders) and control group (normal test) play the negative emotional film and the changes of the two groups of subjects' emotional experience were investigated with the scale of self-aging. The results showed that and compared to normal subjects and anxiety in patients with less by accepted more by inhibition of expression to regulate emotions, and it is this inappropriate emotion regulation strategies use leads to higher in the experimental group subjects were negative experience. In another experiment, Campbell-Sills et al. further confirmed that the reception strategy is a more adaptive emotion regulation strategy with respect to expression suppression [19].

At the same time, Hofmann and others in their study for the first time compared the cognitive reappraisal, the expression of inhibition and reception strategy in the regulation of the effect of anxiety. After causing anxiety in the lab, using the guide language to guide the subjects to use the above three kinds of emotion regulation strategies to reduce anxiety, results show that the use of expression suppression strategy subjects in negative emotion and physiological reaction is the highest; using cognitive reappraisal strategy don't is in negative emotion and physiological responses are the lowest, and use acceptance policy subjects decreased significantly in physiological reaction, and cognitive reappraisal group had no significant difference, but in emotional experience and expression suppression had no significant difference [5]. This means that the adjustment of the strategy to the emotional experience is not ideal, but it can reduce the physiological reaction. This is different from the previous results, the researchers pointed out that the subjects and possible experimental treatment, while the future research needs to make further validation and interpretation.

In summary, the existing research showed that cognitive reappraisal strategy in emotional regulation effect is better than that of reception strategy and expressive suppression, but acceptance policies and inhibited the expression of the two relationships also exist differences, also need further research.

\section{Comparison of the Neural Mechanisms of Cognitive Reappraisal, Expression Inhibition and Acceptance Strategies}

The comparison of the neural mechanisms of the two kinds of emotion regulation strategies, which are cognitive reappraisal and expression inhibition, has been studied. The inner neural mechanism has gradually emerged; but as a new kind of emotion regulation strategy, the relevant research of the reception strategy is less, so the neural mechanism of the adjustment of the strategy is still needed. Based on this, this paper will first introduce the neural mechanism of cognitive reappraisal and expression inhibition, and on the basis of this, we can conclude the neural mechanism of emotion regulation strategies to regulate the emotion, in order to contribute to the future research.

\subsection{The Neural Mechanism of Cognitive Reappraisal}

The cognitive reappraisal strategy of emotion regulation research is found in a reappraisal of negative emotional activation in the dorsal anterior cingulate cortex and prefrontal cortex, also according to the different evaluation goals, reduced enhance and maintained evaluation system such as the amygdala and insula activity [20], shows 
that the prefrontal cortex plays an important part in regulating emotions. Ochsner let the subjects use cognitive strategies to regulate emotion, and the results showed that the activities of the left dorsal lateral prefrontal cortex increased and the activity of the amygdala decreased [21]. In addition to the prefrontal cortex and cognitive reappraisal related activation regions often also include some emotional processing of cortical and subcortical structures, such as amygdala, anterior button back to the cortex, the orbitofrontal cortex [22].

Based on these findings, a number of researchers have proposed the hypothesis that prefrontal cortical areas are associated with the direct and indirect pathways that regulate emotional experience and responses. Direct access hypothesis that cognitive reappraisal success or not directly related to cortical activity, this assumption indicates the reappraisal to a new cognitive explanation of form mainly affecting the cortex system. These cortical systems are associated with changes in cognitive assessment or the assessment of the subjects' emotional state. The indirect pathway assumes that the prefrontal cortex regulates emotional responses and experiences through the mediation of subcortical structures. That is, the cognitive reappraisal of the neural back route consists of two parts: the "top" of the nervous system and the "bottom" of the emotional evaluation and generation system [23].

At the same time, study of wager et al. on the intermediary variable analysis method applied to fMRI data analysis, reveals the complex relationship between the prefrontal cortex and the results. For emotion regulation, there are at least two pathways providing evidence: a positive pathway, which is associated with an increased positive emotional response, and is regulated by the nucleus accumbens/ventral striatum. Another negative pathway, which is associated with an increased negative emotional response, is regulated by the amygdala [24].

\subsection{The Neural Mechanism of Inhibition of Expression}

Current study on the neural mechanism of expression inhibition is few, in general, using expression suppression strategy, sustained activation of the amygdala and insula, areas of the brain. In addition, in contrast to cognitive assessment, the activation of the medial frontal lobe is positively correlated with the response of the peripheral nerve and the activation degree is greatest when the negative emotional reaction is inhibited. Cognitive reappraisal is an early stage strategy, and it reduces the emotion without the need for a time sustained efforts; in contrast, expression inhibition requires effort to restrain caused by strong facial expressions and behavior by emotional stimuli. The study of Goldin finds that, expression suppression only in the late period (10.5 15 seconds) activates some brain regions, including prefrontal cortex occipital temporal lobe, precuneus, occipital region and ventrolateral prefrontal cortex [25].

\subsection{Shared Brain Regions of Emotion Regulation Strategies}

Although the two kinds of control strategies in the process of using, the activation and inhibition degree of amygdala and insula is different, cognitive reappraisal can inhibit the activation of amygdala and insula, and the expression inhibition enhanced the activation of both sides of the amygdala and the frontal island, but both of two activated ventral and lateral prefrontal and lateral orbital frontal cortical regions, suggests that there may be a common cognitive process in the two regulatory strategies, namely, cognitive reappraisal and expression inhibition showed up the separation and sharing of the brain regions. Therefore, there are reasons to infer that the change of the three kinds of emotional regulation to regulate emotional experience and physiological reactions is smaller than the presence of a common brain areas. Although existing studies on the receptive strategies for the neural mechanisms are very few, but we can predict that the primary brain regions of the receiving strategy must also exist in the frontal and lateral orbital frontal cortex. This also requires follow-up researchers to reveal.

5 Summary and Outlook

In the study of emotion regulation strategies, it is focused on the effects of cognitive reappraisal of expression inhibition and reception strategies on the emotional response and the corresponding mechanisms of the brain. To sum up, there are four points:

(1) Cognitive reappraisal, inhibition of expression and reception strategy can reduce the external expression of negative emotions, but the effects of the three on the internal emotional experience and physiological changes are different.

(2) Using cognitive reappraisal to regulate emotion and physiological reaction caused by weakened, sympathetic activation is reduced, parasympathetic activation was enhanced; and the inhibition of the expression of emotion regulation, caused by strong physiological response, sympathetic activation is stronger, and to accept 
the strategy research has found the regulation effect of emotional and response to physiological effects between cognitive reappraisal and expression suppression between, but has yet to future research to experimental measurement and verification.

(3) When people use cognitive reappraisal strategy, and emotional responses are related to the areas of the brain such as the amygdala and the ventral part of the medial orbitofrontal cortex decreased activation, but the strategy of expression suppression in amygdala activation levels did not decrease significantly, and the ventral part of the medial orbitofrontal cortex activation increased, which not only have the brain areas of separation and sharing of brain regions. On the basis of this, the main brain areas of the receiving strategy must also exist in the frontal and lateral orbital frontal cortex areas.

(4) In terms of clinical pathology, long term use of the expression suppression strategy is more likely to produce emotional regulation disorders, and reception strategy can make up for this shortcoming, reception strategy has the potential in clinical use.

We can see that the existing research is mainly focused on the comparison of cognitive re-evaluation, inhibition of expression and reception strategy of negative emotions. But what is the effect of the three on positive emotion regulation? This requires us to further explore, in particular, the behind neural mechanisms and specific implementation process of the three. From this, the following prospects for future research:

(1) The present study is less involved in the direct comparison between the effects of the strategies and cognitive assessment, and the inhibition of expression on the emotional and physiological responses. The third is how to adjust the effect, which needs to be studied in the future.

(2) On cognitive reappraisal and expression suppression neural mechanism research has become more mature, the separation of the two neural mechanism and sharing been widely validated, but received almost no research on the neural mechanism of strategy, it with the traditional regulation strategies (cognitive reappraisal and expression suppression) separation of the neural mechanisms and sharing is in-depth study.

(3) The process of the generation and regulation of emotion is a complex process, the result and significance of using different regulation strategies are different. Thus, the diversity of emotion regulation strategies should be further concerned. At the same time, the existing research limited subjects using only a strategy, but in reality regulation may be used at the same time, this regulation way diversity and synchronized to the future further study.

\section{References}

[1] Lerner, J.S. and Keltner, D. (2000) Beyond Valence: Toward a Model of Emotion-Specific Influences on Judgement and Choice. Cognition \& Emotion, 14, 473-493. http://dx.doi.org/10.1080/026999300402763

[2] Rice, J.A. (2003) The Effects of Emotion Regulation on Children's Memory for Educational Material. University of California, Irvine.

[3] Gross, J.J. (1998) Antecedent- and Response-Focused Emotion Regulation: Divergent Consequences for Experience, Expression, and Physiology. Journal of Personality and Social Psychology, 74, 224. http://dx.doi.org/10.1037/0022-3514.74.1.224

[4] Hayes, S.C. (2004) Acceptance and Commitment Therapy, Relational Frame Theory, and the Third Wave of Behavioral and Cognitive Therapies. Behavior Therapy, 35, 639-665. http://dx.doi.org/10.1016/S0005-7894(04)80013-3

[5] Hofmann, S.G., Heering, S., Sawyer, A.T. and Asnaani, A. (2009) How to Handle Anxiety: The Effects of Reappraisal, Acceptance, and Suppression Strategies on Anxious Arousal. Behaviour Research and Therapy, 47, 389-394. http://dx.doi.org/10.1016/j.brat.2009.02.010

[6] Li, W., Zhang, D.J. and Lei, C.X. (2011) Anxiety Regulation: Acceptance Policy and Expressive Suppression and Reappraisal Strategies. Psychological Exploration New, 4, 372-376.

[7] Wang, Z.H. and Guo, D.J. (2003) Review of the Research on the Process and Strategy of Gross Emotion Regulation. Progress in Psychological Science, 6, 629-634.

[8] Gross, J.J. (2002) Emotion Regulation: Affective, Cognitive, and Social Consequences. Psychophysiology, 39, 281291. http://dx.doi.org/10.1017/S0048577201393198

[9] Gross, J.J. (2001) Emotion Regulation in Adulthood: Timing Is Everything. Current Directions in Psychological Science, 10, 214-219. http://dx.doi.org/10.1111/1467-8721.00152

[10] Huang, M.E. and Guo, D.J. (2001) Study on the Regulation of Emotion Regulation and Depression of College Students. Chinese Journal of Mental Health, 6, 438-441.

[11] Gross, J.J. (2009) Handbook of Emotion Regulation. Guilford Press, New York. 
[12] Gross, J.J. (1998) The Emerging Field of Emotion Regulation: An Integrative Review. Review of General Psychology, 2, 271-299. http://dx.doi.org/10.1037/1089-2680.2.3.271

[13] John, O.P. and Gross, J.J. (2007) Individual Differences in Emotion Regulation. In: Gross, J.J., Ed., Handbook of Emotion Regulation, Guilford Press, New York, 351-372.

[14] Cheng, L., Yuan, J.J., He, Y.Y. and Lee, H. (2009) Emotion Regulation Strategies: Cognitive Reappraisal Is Better Than Expression Inhibition. Psychological Science Progress, 4, 730-735.

[15] Gross, J.J. and Levenson, R.W. (1997) Hiding Feelings: The Acute Effects of Inhibiting Negative and Positive Emotion. Journal of Abnormal Psychology, 106, 95-103. http://dx.doi.org/10.1037/0021-843X.106.1.95

[16] Ray, R.D., Wilhelm, F.H. and Gross, J.J. (2008) All in the Mind’s Eye? Anger Rumination and Reappraisal. Journal of Personality and Social Psychology, 94, 133-145. http://dx.doi.org/10.1037/0022-3514.94.1.133

[17] Huang, M. and Guo, D.J. (2002) The Process of the Change of Emotion Regulation and Response Regulation. Acta Psychologica Sinica, 34, 371-380.

[18] Campbell-Sills, L., Barlow, D.H., Brown, T.A. and Hofmann, S.G. (2006) Acceptability and Suppression of Negative Emotion in Anxiety and Mood Disorders. Emotion, 6, 587-595. http://dx.doi.org/10.1037/1528-3542.6.4.587

[19] Campbell-Sills, L., Barlow, D.H., Brown, T.A. and Hofmann, S.G. (2006) Effects of Suppression and Acceptance on Emotional Responses of Individuals with Anxiety and Mood Disorders. Behaviour Research and Therapy, 44, 12511263. http://dx.doi.org/10.1016/j.brat.2005.10.001

[20] Gong, X., Huang, Y.X. and Luo, Y.J. (2010) Research Progress in the Neural Basis of Emotion Regulation. Chinese Journal of Behavioral Medicine and Brain Science, ISTIC, 19, 2.

[21] Ochsner, K.N., Bunge, S.A., Gross, J.J. and Gabrieli, J.D. (2002) Rethinking Feelings: An fMRI Study of the Cognitive Regulation of Emotion. Journal of Cognitive Neuroscience, 14, 1215-1229. http://dx.doi.org/10.1162/089892902760807212

[22] Ochsner, K.N., Ray, R.D., Cooper, J.C., Robertson, E.R., Chopra, S., Gabrieli, J.D. and Gross, J.J. (2004) For Better or for Worse: Neural Systems Supporting the Cognitive Down- and Up-Regulation of Negative Emotion. Neuroimage, 23, 483-499. http://dx.doi.org/10.1016/j.neuroimage.2004.06.030

[23] Zhao, J. and Shi, X.S. (2010) Emotion Regulation: Process, Influencing Factors and Neural Mechanism. Journal of Liaoning Normal University (Social Science Edition), 6, 39-43.

[24] Wager, T.D., Davidson, M.L., Hughes, B.L., Lindquist, M.A. and Ochsner, K.N. (2008) Prefrontal-Subcortical Pathways Mediating Successful Emotion Regulation. Neuron, 59, 1037-1050. http://dx.doi.org/10.1016/j.neuron.2008.09.006

[25] Goldin, P.R., McRae, K., Ramel, W. and Gross, J.J. (2008) The Neural Bases of Emotion Regulation: Reappraisal and Suppression of Negative Emotion. Biological Psychiatry, 63, 577-586. http://dx.doi.org/10.1016/j.biopsych.2007.05.031 